\title{
"A CFD-based study of steady-state multiplicity in a gas-solid vortex reactor for oxidative coupling of methane"
}

\section{Laurien A. Vandewalle, Kevin M. Van Geem, Guy B. Marin}

The ideal reactor for oxidative coupling of methane (OCM) has two key characteristics: 1) limited species backmixing to maximize the selectivity towards intermediate $C_{2}$ products, and 2) sufficient thermal backmixing to allow steady-state multiplicity and autothermal operation. Non-reactive computational fluid dynamic (CFD) simulations have already shown that the first of these characteristics can be obtained in a gas-solid vortex reactor (GSVR). Whether the GSVR also exhibits the second key characteristic, i.e., sufficient thermal backmixing, is less straightforward to verify. A reactive Euler-Euler CFD methodology is developed for this purpose, allowing to simulate the GSVR while using detailed microkinetic models for both the homogeneous gas phase and heterogeneous surface chemistry. Simulations of a 2D adiabatic GSVR for OCM are performed, for inlet temperatures ranging from $873 \mathrm{~K}$ to $1198 \mathrm{~K}$, in $25 \mathrm{~K}$ increments, while fixing the inlet composition, mass flow rate, catalyst mass and total pressure. The possibility for steady-state multiplicity is assessed by numerically igniting some of the non-ignited steady states, and evaluating whether or not these cases reach a new steady state on the ignited branch. Thanks to steady-state multiplicity, a $\mathrm{CH}_{4}$ conversion of $40 \%$ (see Figure 1) and $\mathrm{C}_{2}$ selectivity of about $75 \%$ (incl. $53 \% \mathrm{C}_{2} \mathrm{H}_{2}$ ) can be obtained on the ignited branch, for an inlet temperature of merely $873 \mathrm{~K}$.

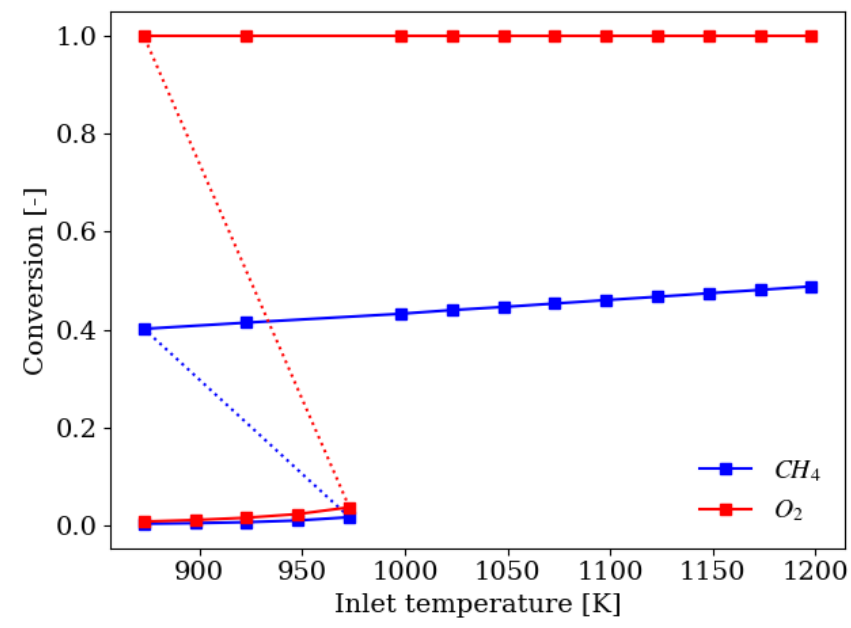

Figure 1: Conversion of $\mathrm{CH}_{4}$ and $\mathrm{O}_{2}$ in a GSVR for OCM, clearly showing steady-state multiplicity. $\left(\mathrm{Sr}-\mathrm{La} / \mathrm{SiC}\right.$ catalyst, $\left.\mathrm{P}=2 \mathrm{bar}, \mathrm{m}_{\mathrm{cat}} / \mathrm{F}^{0} \mathrm{V(NTP), \textrm {CH } 4}=3.6 \mathrm{~kg} . \mathrm{s} / \mathrm{Nm}^{3}, \mathrm{CH}_{4}: \mathrm{O}_{2}=4\right)$ 